\title{
The Impact of Link Suggestions on User Navigation and User Perception
}

\author{
Ion Juvina ${ }^{1}$ and Eelco Herder ${ }^{2}$ \\ ${ }^{1}$ Institute of Information and Computing Sciences, Utrecht University \\ Padualaan 14, De Uithof, 3584 CH Utrecht, The Netherlands \\ ionecs.uu.nl \\ ${ }^{2}$ Department of Computer Science, University of Twente \\ P.O. Box 217, 7500 AE Enschede, The Netherlands \\ herder@cs.utwente.nl
}

\begin{abstract}
The study reported in this paper explores the effects of providing web users with link suggestions that are relevant to their tasks. Results indicate that link suggestions were positively received. Furthermore, users perceived sites with link suggestions as more usable and themselves as less disoriented. The average task execution time was significantly lower than in the control condition and users appeared to navigate in a more structured manner. Unexpectedly, men took more advantage from link suggestions than women.
\end{abstract}

\section{Introduction}

For many people, the web has become a major source of information. More and more people primarily use the web for private matters such as planning their holidays, deciding between products and many other activities.

In contrast to most desktop applications, web sites generally are designed for a general audience with varying goals [14]. As it is hard to satisfy all categories of users with one design, adaptive hypermedia systems try to better support the users by personalizing content or link structure. Traditional techniques in the latter category involve link hiding, sorting, annotation, direct guidance and hypertext map adaptation [2]. When trying to find information related to a task, users have to rely on proximal cues such as the link anchor text to decide what their next action will be [10]. If the proximal cues are not clear enough, or if the users do not have sufficient insight on the structure of the site, they may become disoriented, i.e. they don't know their current position in a web site, how they came to that point or where to go next [4]. Various studies have been carried out to infer user goals from their actions [e.g. 3]. Given these goals, the utility of the various navigation options on a web page can be estimated [7][12] and communicated to the user by means of link relevancy indicators, or link suggestions.

While user-adaptive systems appear to be a good idea, it still is an open issue how the benefits from adaptations can be evaluated [17]. We conducted a user study in which participants were asked to carry out several predefined everyday tasks. In one 
condition, the participants were provided with predefined link suggestions. Various indicators of user's behavior and perception were measured. We found evidence that link suggestions based on the user's goals have a positive impact: they cause the users to navigate in a more structured way, which makes them less vulnerable to disorientation [4].

The remainder of this paper is structured as follows. In the next section we present our research questions. We continue with the setup of the experiment. After presenting the results, we conclude with a short discussion.

\section{Individual Differences, Disorientation and Navigation Styles}

There is a vast amount of literature on individual differences in web navigation. In a previous study we found spatial ability and domain expertise to be the most important determinants of user performance in web tasks [6]. It has been shown that there are differences in favor of men with regard to spatial ability [15], web searching behavior, and learning performance [13]. Women are more likely to use a rote way-finding strategy - attending to instructions on how to get from place to place - whereas men are more likely to report to use an orientation strategy - maintaining a sense of their own position in relation to environmental reference points [15]. For this reason, we expect women to benefit from navigation support that supports the rote way-finding strategy, more so than men, thus compensating for the so-called 'gender gap' in web use.

As mentioned in the introduction, disorientation is a major issue in web navigation that is mainly caused by the non-linearity of web sites; on each page, users have to decide between alternative options, which includes following links or backtracking to pages visited earlier. Although the problem has been given the label 'disorientation', it is hard to measure or to quantify. Ahuja and Webster [1] developed a questionnaire that is shown to indicate a user's perceived disorientation. Various attempts have been made to relate patterns in user navigation, most importantly patterns related to page revisits, to success measures and disorientation [5][8][16]. In a previous study [4] we found a weak navigation style that was associated with perceived disorientation.

Based on these previous findings, we formulated the following hypotheses to guide the study:

1. Link suggestions will generally be well received.

2. Link suggestions improve perceived usability and reduce disorientation, as experienced by the users.

3. Link suggestions will influence the way users navigate; the differences can be interpreted as an argument in favor of providing support.

4. Women will benefit more from links suggestions than men. 


\section{Experimental setup}

In order to check for differences caused by link suggestions to users perceptions and navigation behavior an experimental approach was employed, which is described in this section.

\subsection{Web Navigation Tasks}

First matter of concern for the experimental setup was triggering realistic web navigation behavior. Five web navigation tasks were created based on the collection of cases presented in [9], following suggestions from [7]. An example task is presented in figure 1.

Participants were instructed to start each task at a specified website's home page. They were allowed to use other websites than the indicated ones; the only restriction was to start at the specified websites.

This summer you will spend a long weekend in London with your girl/boy friend. Both of you would like to visit the top attractions and some museums. But, most importantly, you want to visit one of the great musicals in West End.

Given Facts:

- You already have plane tickets to Heathrow Airport

- You still have to book a hotel, preferably near the West End theatre district

- You have sufficient money to spend during the weekend

To do:

Go to http://www.visitlondon.com and find answers to the following questions:

- Find a small hotel in the West End district

- How do you get from Heathrow Airport to the city center?

- In what theatre does the Lion King play?

- Find a restaurant in Covent Garden that offers pre-theatre menus.

Figure 1. An example web navigation task

\subsection{Experimental Manipulation}

Various strategies for generating link suggestions can be thought of. The simplest case is when the content providers indicate the most important sections of the content from their point of view. As users may have different goals for visiting a site, it might be a better idea to provide link suggestions that match the current context of use. In this experiment we provided users with suggestions that are relevant to their tasks.

Suggestions were generated based on simulations of a cognitive model similar in principle to CoLiDeS, a cognitive model of web navigation presented in [7]. CoLiDeS uses Latent Semantic Analysis (LSA) to calculate the semantic similarity between goal description and the available links on the current webpage. The link most similar in description to the task goal is selected to be clicked on. User behavior was simulated 
in advance based on the task descriptions. Semantic similarities between these task descriptions and the texts of the links leading to task solutions were calculated with LSA.

For each task, one or more successful paths were generated. In the navigation support condition, links on these paths were highlighted to the participants - see figure 2. In the control condition, participants executed the same tasks without any support. The link suggestions were generated on the fly using the Scone framework for development and evaluation of web enhancements [18].

Participants in the support condition were instructed that suggestions were automatically generated by a cognitive robot, they were meant to help participants in doing their tasks, and they could be followed or not. Participants got suggestions only when they arrived at specific pages.

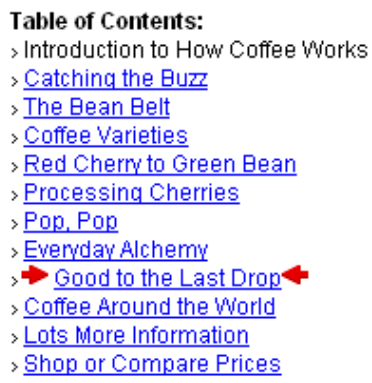

Figure 2. Example of link suggestion: the two red arrows point at (suggest) the link text "Good to the last drop".

\subsection{Participants}

Thirty-two participants, mainly students of various studies at Utrecht University, were recruited with advertisements. To qualify for participating, a minimum level of English language skills and Internet experience was required. The participants were randomly assigned to one of the two conditions - sixteen participants in each condition.

\subsection{Measures of User Navigation and User Perceptions}

Several measures on navigation complexity and patterns of page revisits were calculated. For matters of brevity we limit ourselves to describing the most relevant measures in the context of this study and refer to [4][5] for a more complete overview. The meaning of these measures is described below and illustrated in figure 3 .

- $\quad$ back button usage is the percentage of back button clicks among the navigation actions;

- $\quad$ the relative amount of home page visits is the number of visits to the web pages that the participants used to start the different tasks, divided by the total number of page visits;

- compactness [8] indicates that users follow a 'shallow' search strategy;

- $\quad$ the navigation stratum [8] is a measure designed to capture the linearity of user navigation;

- $\quad$ the average connected distance indicates the average distance between any two pages in a navigation path. In short, it indicates how confident users are that they 'will find their way back later' [4]. 

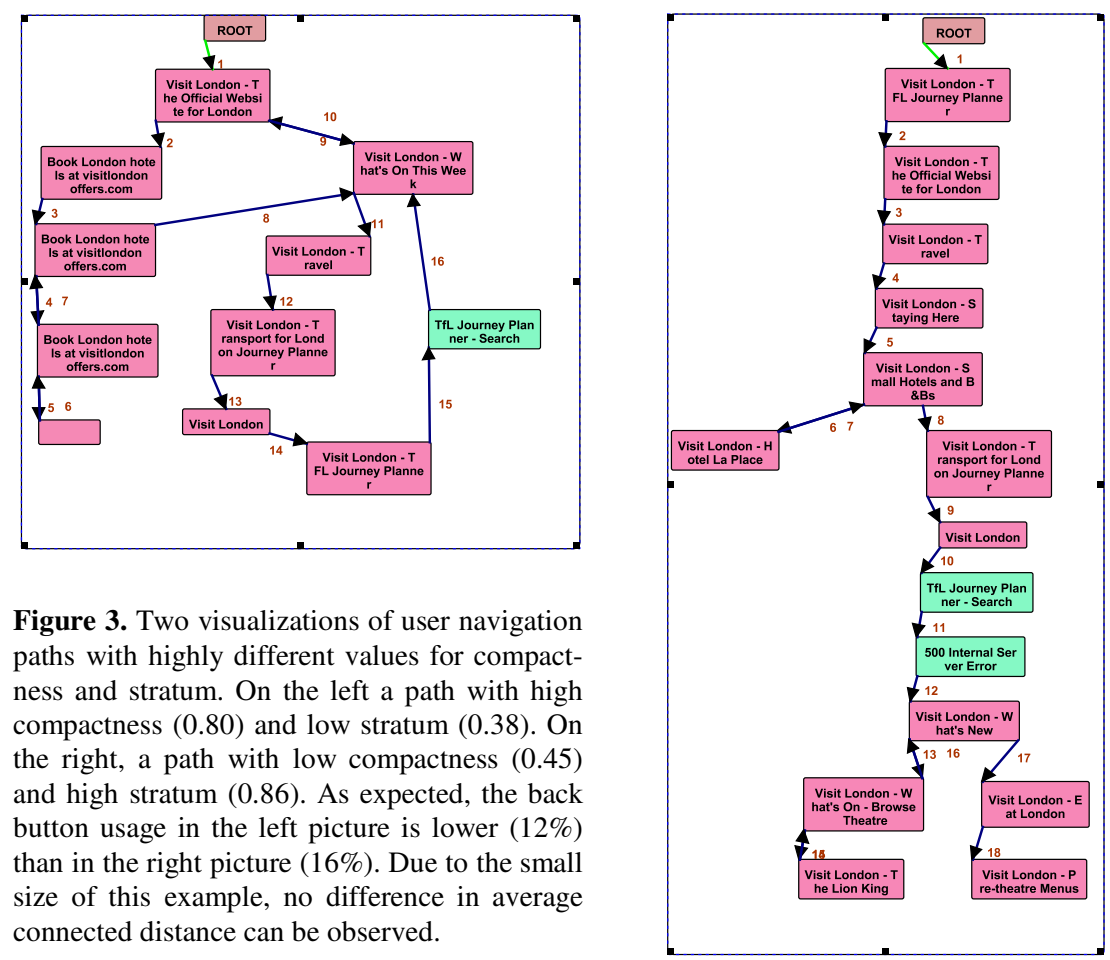

Figure 3. Two visualizations of user navigation paths with highly different values for compactness and stratum. On the left a path with high compactness (0.80) and low stratum (0.38). On the right, a path with low compactness $(0.45)$ and high stratum (0.86). As expected, the back button usage in the left picture is lower (12\%) than in the right picture $(16 \%)$. Due to the small size of this example, no difference in average connected distance can be observed.

A post-navigation questionnaire was used to measure user opinions on usability of the websites used and the users' perceived disorientation [1]. For each item of the questionnaire a 5-point Likert scale from 'strongly disagree' to 'strongly agree' was used. The 16 participants in the support condition were given four additional items on how they perceived the provided suggestions:

- The suggestions given by the robot were helpful

- I felt the suggestions were intrusive / annoying

- I believed I could trust the suggestions given by the robot

- I felt being manipulated by the given suggestions

The variable gender was added as an independent variable in the analysis phase to check whether it interacts with the fixed factor (support). The duration of each session was 55 minutes, of which 40 minutes were spent on carrying out the navigation tasks.

\section{Results}

In this section we present the results of the study described above. We start with the participants' opinions on link suggestions. Then we describe the impact of link sug- 
gestions on user perceptions and task execution time. We continue with the influence of link suggestions on user navigation behavior. We conclude with a brief look at gender differences.

\subsection{Link Suggestions Are Positively Received}

Table 1 shows the number of participants expressing their agreement or disagreement with each of the four questionnaire items concerning the way suggestions are perceived. It can be observed that most participants (13) do not perceive suggestions as intrusive, annoying or manipulative. A relatively high number of participants (11) trusted link suggestions; but there is no clear evidence that the suggestions are perceived as useful.

Table 1. User perception of link suggestions

\begin{tabular}{|l|c|c|c|}
\hline & Disagree & Neutral & Agree \\
\hline Suggestions were helpful & 5 & 4 & 7 \\
\hline Suggestions were intrusive / annoying & 13 & 1 & 2 \\
\hline I could trust suggestions & 4 & 1 & 11 \\
\hline I felt being manipulated by the given suggestions & 5 & 4 & 7 \\
\hline
\end{tabular}

\subsection{Suggestions Improve User Perceptions and Decrease Task Execution Time}

Participants in the support condition disagreed to a larger extent than participants in the control condition with the following statements: 'It was difficult to find the information I needed on these sites' $(\mathrm{t}=-2.72, \mathrm{p}=0.01)$, and 'Labels of links and categories confused me' $(\mathrm{t}=-2.83, \mathrm{p}=0.008)$. Participants receiving suggestions agreed at a larger extent than participants in the control group that 'the websites can be used without previous experience' $(\mathrm{t}=2.33, \mathrm{p}=0.027)$. For all other items, differences were not significant.

When looking at aggregated measures of user perceptions - perceived disorientation and perceived usability - the differences between conditions appear to be nonsignificant. However, there is a marginally significant result: the level of disorientation is lower in the support condition, but this difference is significant only at an alpha level of 0.10 (two tailed). We also observed a significant interaction between the variable gender and the variable support in relation to perceived disorientation $(\mathrm{F}=5.12$, $\mathrm{p}=0.032$ ); men and women benefit to different extents from link suggestions. This last result will be dealt with at the end of this section. When the interaction between gender and support is taken into consideration, the effect of support becomes significant $(\mathrm{F}=9.43, \mathrm{p}=0.005)$. Therefore, it is now clear that there is a significant effect of providing suggestions on perceived disorientation, but only for men.

When the two conditions are compared based on the average task execution time, a significant difference is revealed - see table 2. On average, participants in the control condition spent 558 seconds per task. In the support condition, the average time spent per task is 391 seconds $(\mathrm{t}=5.99 ; \mathrm{p}<0.01)$. The spread of task execution times in the 
support condition is almost twice as low as in the control condition. This difference in variance between the two groups is a natural consequence of our manipulation; the aim of link suggestions is to prevent users from spending time on unsuccessful trials.

Table 2. Task execution times per condition

\begin{tabular}{|l|l|l|l|}
\hline & N & Mean & Std. Dev. \\
\hline Control & 16 & 558,22 & 99,93 \\
\hline Support & 16 & 390,98 & 50,05 \\
\hline
\end{tabular}

\subsection{User Navigation Is Better Structured}

The results presented in the previous subsections indicate that participants did believe that the link suggestions could be trusted and that they were considered slightly helpful. We now turn to the question whether the link suggestions actually changed the participants' approach to solving the tasks at hand.

As explained in section 3.4, we extracted a number of measures from the navigation paths that captured patterns of page revisits, page view times and navigation complexity [4][5]. An independent-samples t-test was carried out to find significant differences in means between the two conditions. The result showed that participants in the support condition:

- $\quad$ used the back button less $(\mathrm{t}=-2.24, \mathrm{p}=0.03)$;

- the navigation paths had a lower compactness $(\mathrm{t}=-3.02, \mathrm{p}=0.005)$ and a higher stratum $(\mathrm{t}=3.42, \mathrm{p}=0.002)$, i.e. the paths were more linear;

- the average connected distance in the navigation path was higher $(\mathrm{t}=2.26$, $\mathrm{p}=0.031$ )

We also carried out principal component analysis on the twenty-two measures with equamax rotation to find linear combinations of the measures that indicate navigation patterns. Four factors were found that were quite similar to the four factors found in a previous study [5]. We will concentrate here on the fourth factor, of which the means differed significantly between the two conditions $(\mathrm{t}=-4.01, \mathrm{p}=0.000)$. This factor correlates with $(\mathrm{p}<0.05)$ :

- $\quad$ high compactness $(\mathrm{r}=0.896)$ and low stratum $(\mathrm{r}=-0.861)$

- $\quad$ many visits to the site's home page $(r=0.496)$,

- $\quad$ short average connected distance $(\mathrm{r}=-0.388)$

- $\quad$ frequent use of the back button $(\mathrm{r}=0.361)$

Apparently, the link suggestions caused the participants to navigate in a more linear manner and reduced the number of visits to the site's home pages. There are two possible explanations for this effect: either the participants simply followed the suggested links, without bothering to explore the site structure [8] - a negative effect, or the participants got stuck less often - getting stuck usually results in returning to the site's home page to start another trial [11] - which is a positive effect.

Analysis of the navigation path visualizations led us to the firm belief that the latter is the case - see figure 4 . In three of the five tasks the participants that were not provided with link suggestions typically appeared to randomly return to pages visited 
Figure 4. In the picture on the right the navigation path of a participant working on a task without link suggestions is displayed. The randomness of page revisits and the amount of visits is clearly visible. In the picture below the navigation path of a participant working on the same task, but with link suggestions, is displayed.

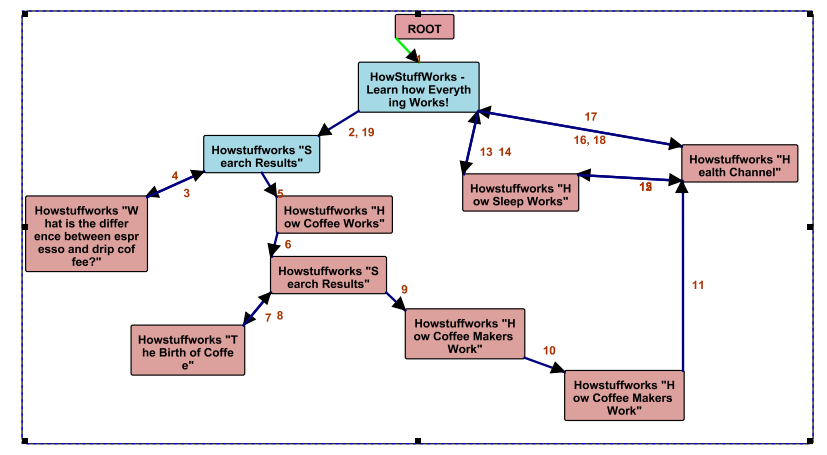

before and eventually return to the site's home page for another trial - effects that are frequently reported to be caused by disorientation [11][16].

There is no significant interaction between the variables gender and support with respect to any of the navigation measures considered. In other words, navigation patterns are basically the same for men receiving link suggestions as for women receiving link suggestions.

\subsection{Winners Win Even More - Gender Differences}

In general, when all participants from both conditions were pooled together, no differences between men and women were found in this study, at least with respect to the variables we have considered here - i.e. navigation measures and user perceptions.

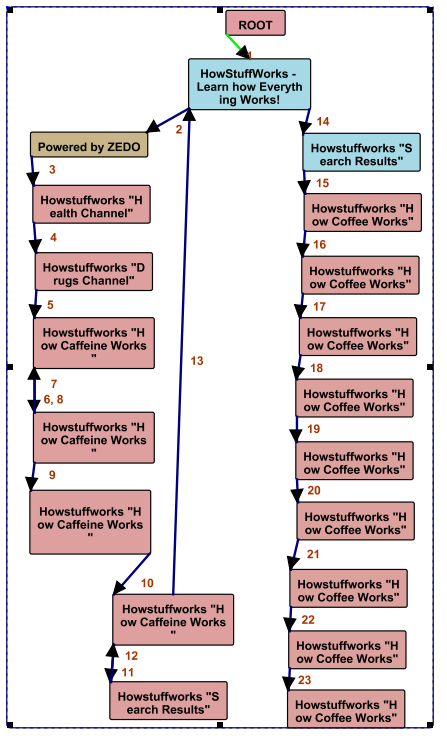

However, although in the control condition women and men declare about the same level of disorientation, in the support condition men declare a much lower level of disorientation than women. Women do not seem to benefit from being provided with navigation support, their perceived disorientation levels are about the same in the 2 conditions. In the support condition, men and women also differ with respect to perceived usability. Men receiving navigation support perceive the websites more usable than men not receiving support $(\mathrm{t}=-2.66, \mathrm{p}=0.029)$. The difference is not significant for women.

Therefore, there seems to be a gender gap indeed, but in this study we were able to find it only with respect to how much the two genders are able to benefit from being provided with navigation support and how they perceived the usability of the systems they used when such support was offered. 


\section{Discussion and Conclusion}

In this study we explored the impact of providing link suggestions on user navigation behavior and on user perceptions. In general, highlighting links that are relevant to the task at hand is a well-received navigation support. Link suggestions make navigation path more linear, more structured and less redundant - a style that is associated with low degrees of perceived disorientation. Users provided with link suggestions were expected to perceive websites as more usable and themselves as less disoriented, but this expectation was confirmed only for men. Women seem to profit from suggestions only objectively - their navigation path becomes more structured, but not subjectively - their perceptions do not improve when receiving link suggestions. A possible explanation for this effect is that men use the link suggestions in addition to the available orientation clues.

There are a number of limitations to this study. First, we have not yet evaluated the impact of link suggestions on task performance. So far we only know that link suggestions make users' navigation more structured, reduce task execution time and improve user perceptions, but we don't know whether they actually help users. However, the lack of task performance results is not that important, as the impact of link suggestions on task performance varies per usage context and the way link suggestions are generated. Second, the number of female participants exceeded more than twice the number of male participants ( 22 female, 10 male) due to the way participants were recruited.

In our study we explicitly attempted to let users carry out real-life tasks. This aim is obviously violated by the fact that the participants were given predefined tasks - or scenarios - in a laboratory setting. Although we have the impression that the impact of the artificial context is quite low, it is an open question what the effect of link suggestions will be in real-life situations in which users work on multiple tasks simultaneously. Nevertheless, before being able to observe the effects 'in the wild' it is necessary to first study them in more controlled settings.

In conclusion, link suggestions relevant to users' tasks have in general a positive impact on users. However, due to individual differences between users, the effects might not be the same for all users. Moreover, these differences might lead to other possibly counter-intuitive - effects than anticipated. More research and user studies on the effects of personalization techniques are needed to find out what techniques are best suitable for various personalization goals.

\section{Acknowledgements}

The second author's work is carried out in the context of the PALS project, which is sponsored by the Dutch innovative Research Program IOP-MMI. 


\section{References}

1. Ahuja, J.S. and Webster, J. Perceived disorientation: an examination of a new measure to assess web design effectiveness. Interacting with Computers, 14(1), 2001, 15-29.

2. Brusilovsky, P. Adaptive Hypermedia. User Modeling and User-Adapted Interaction 11, 2001, 87-110.

3. Chi, E.H., Rosien, A., Supattanasiri, G., Williams, A., Royer, C., Chow, C., Robles, E., Dalal, B., Chen, J. and Cousins, S. The Bloodhound Project: Automatic Discovery of Web Usability Issues using the InfoScent Simulator. Proc. CHI 2003, 2003.

4. Herder, E. and Iuvina, J. Discovery of Individual User Navigation Patterns. Proc. Workshop on Individual Differences in Adaptive Hypermedia, held at AH2004. 2004, 40-49.

5. Herder, E. and Van Dijk, E.M.A.G. Site Structure and User Navigation: Models, Measures and Methods. Adaptable and Adaptive Hypermedia Systems. ISBN 1-59140-536-X, 2004, 19-34.

6. Juvina, I., \& van Oostendorp, H. Individual differences and behavioral aspects involved in modeling web navigation. Proc.User Interfaces for All, 2004.

7. Kitajima, M., Blackmon, M.H., \& Polson, P.G. A Comprehension-based Model of Web Navigation and Its Application to Web Usability Analysis. People and Computers XIV, Springer: 2000, 357-373.

8. McEneaney, J.E. Graphic and numerical methods to assess navigation in hypertext. Intl. Journal of Human-Computer Studies, 55, 2001, 761-786.

9. Morrison, J. B., Pirolli, P., and Card, S. K A taxonomic analysis of what World Wide Web activities significantly impact people's decisions and actions, UIR Technical report UIR-R2000-17, 2000.

10. Olston, C. \& Chi, E.H. ScentTrails: Integrating Browsing and Searching on the Web. ACM Trans. On Computer-Human Interaction 10 (3), 2003, 177-197.

11. Otter, M. and Johnson, H. Lost in hyperspace: metrics and mental models. Interacting with Computers, 13(1), 2000, 1-40.

12. Pirolli, P., \& Fu, W.-T. SNIF-ACT: A Model of Information Foraging on the World Wide Web. Proc. User Modeling 2003, 2003.

13. Roy, M., \& Chi, M.T.H. Gender Differences in Patterns of Searching the Web. Journal of Educational Computing Research 29, 2003, 335-348.

14. Shneiderman, B.: Designing Information-Abundant Websites: Issues and Recommendations. International Journal of Human-Computer Studies 47 (1). Academic Press, 1997.

15. Sjolinder, M., Individual differences in spatial cognition and hypermedia navigation, Towards a Framework for Design and Evaluation of Navigation in Electronic Spaces. 1998, Swedish Institute of Computer Science.

16. Smith, P.A. Towards a practical measure of hypertext usability. Interacting with Computers, 8(4), 1997, 365-381.

17. Weibelzahl, S., Lippitsch, S. \& Weber, G. Advantages, opportunities, and limits of empirical evaluations: Evaluating adaptive systems. Künstliche Intelligenz 3 (02), 2002, 17 20.

18. Weinreich, H., Buchmann, V. \& Lamersdorf, W. Scone: Ein Framework zur evaluativen Realisierung von Erweiterungen des Webs. Proceedings KiVS 2003, 31-42. 